\title{
Las Islas Galápagos del Ecuador como potencia turística
}

\section{(c) $\frac{10(9)(2)}{\mathrm{BY} N \mathrm{NA}}$}

The Galapagos Islands of Ecuador as a tourist power

Pablo Homero Velasteguí López. ${ }^{1}$

Recibido: 05-12-2018 / Revisado: 07-12-2018 /Aceptado: 08-12-2018 / Publicado: 04-01-2019

Resumen.

DOI: https://doi.org/10.33262/concienciadigital.v2i1.926

El objetivo de este trabajo es dar a conocer que las islas Galápagos del Ecuador como potencia turística internacional por la cantidad de flora y fauna endémica del sector por lo mismo contiene una gran cantidad y calidad, para diferentes clases de turismo como de aventura, naturaleza, sol y playa, científico, gastronómico, y por ende Galápagos fue declarado por la UNESCO "Patrimonio Natural de la Humanidad "en 1978, Galápagos en la actualidad es una de las potencias turísticas de masas más importantes por lo cual le convierte en uno de los puntos turísticos más importantes del Ecuador generando 241.800 visitan en el 2017 de los cuales el 69\% son del extranjeros (167 mil turistas), y turistas nacionales solo el 31\% (75 mil turistas), la distribución se ha mantenido por los últimos 3 años, nadie se imaginó que las islas Galápagos se convirtieran en uno de los destinos de turismo natural más importantes a nivel internacional aportando cientos de millones de dólares a la economía del país que ayudaría a exceder las tasas. Ayudando a general trabajo ya sea de forma directa o indirecta a la población, pero todo esto no es solo un beneficio sino también muy perjudicial para la el sector ya que el turismo masivo puede traer daños grabes en la flora y fauna endémica que hay en sector e incluso a la extinción es decir que desaparezca totalmente del ecosistema, también debemos ver los daños que causa el hombre el cual sería por contaminación o provocando la erosión de la tierra.

Palabras claves: Economía, Galápagos, flora, fauna, directa, indirecta, tipos de turismo.

\footnotetext{
${ }^{1}$ Ciencia digital, Ambato, Ecuador, pablohomerovelastegui@cienciadigital.org
} 


\section{Abstract.}

The objective of this work is to make known that the Galapagos Islands of Ecuador as an international tourism power for the amount of flora and fauna endemic to the sector, therefore it contains a large quantity and quality, for different kinds of tourism such as adventure, nature, sun and beach, scientific, gastronomic, and therefore Galápagos was declared by UNESCO "Natural Heritage of Humanity" in 1978, Galapagos is currently one of the most important mass tourism powers which makes it one of the Ecuador's most important tourist attractions generating 241,800 visitors in 2017 of which $69 \%$ are from foreigners (167 thousand tourists), and national tourists only 31\% (75 thousand tourists), the distribution has been maintained for the last 3 years, nobody imagined that the Galapagos Islands became one of the most important natural tourism destinations internationally, contributing hundreds of millions of dollars to the economy of the country that would help to exceed the rates. Helping general work either directly or indirectly to the population, but all this is not only a benefit but also very damaging to the sector since mass tourism can bring gravest damage to the endemic flora and fauna that exists in the sector and even to extinction is to say that it disappears completely from the ecosystem, we must also see the damages caused by man, which would be caused by pollution or causing the erosion of the earth.

Keywords: Economy, Galapagos, flora, fauna, direct, indirect, types of tourism.

\section{Introducción.}

\section{Turismo en las Islas Galápagos:}

Según (Amador. E, 1996; Barriga.A, 2014; Muñoz.A, 2015 ) El turismo tiene ventajas y desventajas para las Galápagos. Algunas de las partes buenas son que los turistas traen dinero a las islas y son una fuente de ingresos para muchas Galapagueños, sin embargo, también hay partes malas, a medida que más turistas visitan las islas, necesitarán más lugares donde quedarse, es posible que llegará un tiempo en que hay que comprar grandes hoteles que posiblemente pondrán en peligro la vida silvestre cerca, uno de los mayores beneficios que el turismo aporta a la isla es el dinero. Los visitantes aportan una gran cantidad de dinero a la economía Islas Galápagos, las Islas ahora generan más que US\$143 millones al año a través del turismo. Además, más de dos mil personas están empleadas en la industria del turismo. Está formado por 13 islas grandes, 6 pequeñas y 107 rocas e islotes, la Reserva Marina de Galápagos, la segunda más grande del mundo, contienen el último arrecife de coral, sus aguas poseen la mayor biomasa de tiburones del planeta y reciben la única migración de tiburones ballena en estado de gestación”, con el fin de proteger 
al tiburón martillo, una especie en extinción". Las Islas Galápagos no es un destino cualquiera, se trata del mejor destino que puedes elegir para pasar unas extraordinarias vacaciones. No dejes pasar la oportunidad de conocer las maravillas de este fascinante lugar.

Figura N 1: Arribo de turistas en las islas Galápagos

Arribos turísticos a Galápagos 2008-2017

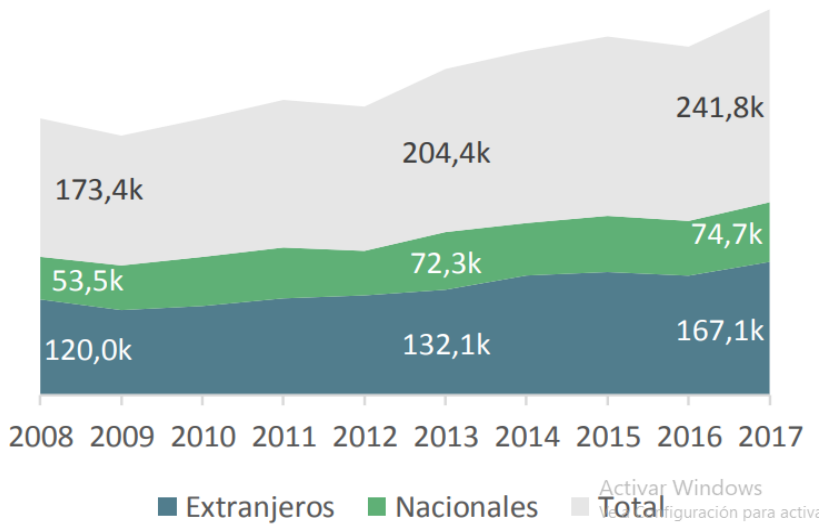

Fuente: Ministerio del Turismo.

\section{Criterio:}

En este proceso ya no solo los profesionales en turismo se pueden dedicarse a la actividad turística en el sector,ya que en la actualidad existen operadoras de turismo y los turistas también contribuyen directamente a las islas, donando proyectos de conservación en todo Galápagos, las tarifas que ofrecen en el parque nacional puede llegar ser \$ 100 por adultos y $\$ 50$ para los niños precio que se han conservado desde el 2014, este impuesto apoya a una variedad de organizaciones de todo el archipiélago, el turismo es la fuente más importante del sector por el simple hecho que el llevar las cosas como los productos básicos y de primera necesidad en adición a los aspectos positivos, el turismo en general los mayores visitantes en el sector son los turistas extranjeros cuando comienzan sus vacaciones, generando trabajo y economía al sector por el hospedaje, alimentación, visitas a áreas protegidas, parque acuáticos, turismo de aventura, guianzas o por la forma indirecta la que sería por personas que ofrecen algún producto extra a lo de turismo, es lo que ayuda a la economía local mediante la compra de los productos ofertados ahora con la gran cantidad de turistas que 
visitan las islas que los agricultores y los pescadores locales no pueden mantenerse al día con la demanda por los turistas.

\section{Características de la Economía del Turismo en las Islas Galápagos del Ecuador.}

Según (Epler.B,2007; Grenier. C, 2017; Portland. P,2017) en su libro de conservación contra natura las islas Galápagos, define que el turismo masivo en las islas comienza cuando Charles Darwin comienza su estudio de las especien en el año de 1831 en una travesía llamada Beagle que duraría cinco años y así logrando recolectar miles de especies las que llevaría a su país para ser investigadas con los mismo que pudo realizar su investigación la hoy reconocida. Entre los años 80 y 90, circunstancias atenuantes como la caída de la economía nacional y disturbios políticos, también motivaron la llegada de ecuatorianos desde la parte continental del país, por este hecho la economía del Ecuador se desplomo cuando en precio del oro negro es decir del petróleo, el principal producto de exportación que daba al país una gran cantidad de dinero se desplomaría y así llevando a la desaparición del sucre y trayendo la dolarización. Poniendo de lado todos los costos de agencias de viajes, los barcos grandes recibieron $\$ 358$ por cada pasajero por la noche, mientras que los más pequeños retuvieron aproximadamente $\$ 310$ po cada noche. Una sociedad sostenible en términos económicos es aquella en la que el uso de manera justa y eficiente de los recursos implica que la gente tenga ganancias económicas, esto se traduce en que la economía de un país, región o pueblo será capaz de sostenerse por sí misma, muchas personas trabajan en las islas la industria turística. Los turistas traen dinero a las islas y lo que gastan es utilizado por la gente del archipiélago para comprar otros productos (como alimento) y servicios, a este intercambio de dinero por productos y servicios es lo que se conoce como economía.

\section{Criterio:}

Se puede decir que la economía de las Islas Galápagos del Ecuador en su mayoría depende del turismo nacional e internacional ya que del mismo se puede generar empleos o trabajos directos (personas que se dedican a la hotelería y al turismo) e indirectamente (restaurantes, vendedores ambulantes) es decir que ambos generan economía a la población del sector, todos estos factores ayudan incluso a las divisas del país, se puede decir que el 3 ingreso económico al país no económico es el turismo ya que es considera como una fábrica no contaminante por los servicios que la misma ofrece turismo natural, aventura o el mejor 
ejemplo del mismo es el ecoturismo, al país lo que daría el ingreso al dólar y con ellos una gran cantidad de turistas que manejaban esta moneda y provocando la nueva manera de ingreso al país que sería el turismo natural, gastronómico y playero las potencias más importantes en ese entonces, en cambio a comenzó del siglo 20 y 21 todo eso cambiaria de forma radical crecimiento de la población la misma que trajo la existencia y nueva creación de sitios turísticos como hoteles, restaurantes y sitio de distracción tanto como para la mañana y noche, la economía de las Islas Galápagos en la actualidad su mayor fuente económica es el turismo no solo por los diferentes recursos turísticos que ofrece sino por la diferente cultura que tiene, el turismo ofertado por medios naturales e implementados los mismos que los hacen llamativos para todos los turistas.

Tabla N 1: Barcos grandes con diferencia a otros barcos

\begin{tabular}{|c|c|c|c|}
\cline { 2 - 4 } \multicolumn{1}{c|}{} & Barcos Grandes. & Otros Barcos. & Todos los Barcos. \\
\hline $\begin{array}{c}\text { Número de } \\
\text { noches } \\
\text { pasajeros }\end{array}$ & 165.671 & 197.555 & 363.226 \\
\hline $\begin{array}{c}\text { Promedio } \\
\text { Precio / } \\
\text { Noche }\end{array}$ & $\$ 365$ & $\$ 310$ & $\$ 333$ \\
\hline $\begin{array}{c}\text { Ganancias } \\
\text { Totales }\end{array}$ & $\$ 59.310 .218$ & $\$ 61.242 .050$ & $\$ 120.552 .268$ \\
\hline
\end{tabular}

Nota. - Se puede demostrar la difieren en ganancia de cada uno de ellos con un $51 \%$ de ganancias superiores.

Fuente: adaptada de conservación contra natura las islas Galápagos. 


\section{Criterio:}

Se puede decir que las grandes embarcaciones por los precios un poco más elevados que los demás barcos no generan tanto dinero ni cruceros por las islas ya que prefieren un poco más lo económico y las actividades que los mismos ofertan.

\section{Flora endémica de las islas Galápagos:}

Según (Arias.I, 2004; Tye.A, 2006; Alvarado.A, 2016) en su informe aclara que en el archipiélago existen 220 especies de plantas endémicas, 399 nativas y 119 introducidas; cabe recalcar que la flora introducida fue trasportada por los viento, el mar, el hombre o por aves que emigraron desde el continente llevado en sus plumas, patas y picos algunas semillas y esporas que pudieron germinar en el suelo insular, provocando las modificaciones, con el trascurrir del tiempo, su propia estructura y fisiología. En la investigación que ha realizado, no hay ninguna especie de gimnosperma nativa en Galápagos la razón es que las esporas de las pteridofitas son fácilmente transportadas a través de grandes distancias por el viento y el continuo contacto genético con las poblaciones continentales disminuye la posibilidad de evolución de nuevas especies y taxones infra específicos por aislamiento geográfico.En la investigación dice claramente que las islas Galápagos sin muy importantes ya que estas contienen una gran variedad de flora endémica ya sea introducida y que evoluciono correctamente para poder adaptarse en la nueva fuente de vida a la que fue otorgada desarrollándose y convirtiéndose en una planta única a nivel global en la isla Santa Cruz, tiene una distribución muy reducida, las cabras y los asnos salvajes son un grave problema para su sobrevivencia.

Figura N 2: flora endémica de las Islas Galápagos del Ecuador

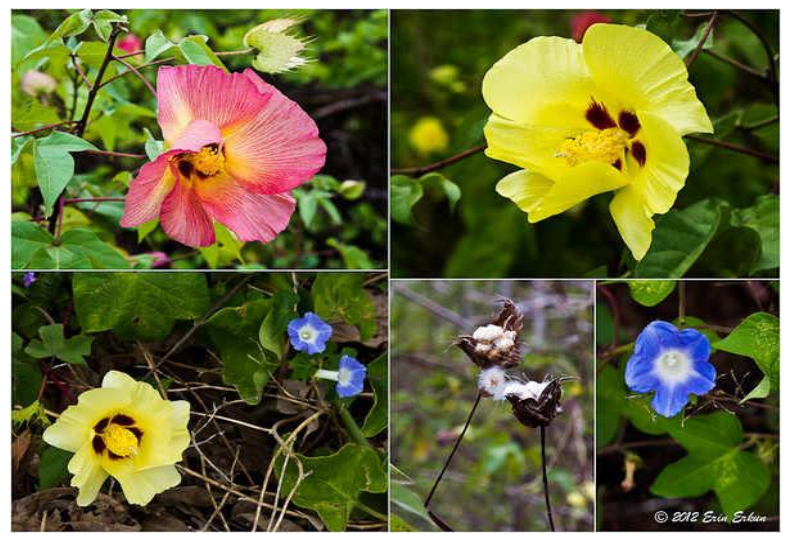

Fuente: Elaboración propia 


\section{Criterio:}

Los tres autores concuerdan con que la flora única de las islas debe ser cuidad, protegida y apresada ya que por ella se realizan estudios que son beneficios para los seres humanos, que con tan solo la pérdida o desaparición de una seria algo catastrófico para el ecosistema, las islas Galápagos merecen la atención en especial porque tienen flora endémica que deben ser cuidadas y protegidas no solo por el ministerio del ambiente sino por todas la personas nativas pero en especial por los turistas que llegan ya que tienen que entender que si solo una planta más desaparece el ecosistema seria afectado gravemente, el endemismo y la radicación y adaptación de las plantas a sus nuevos entornos,

\section{Clases de flora endémica de las islas Galápagos:}

Tabla N 2: Plantas en peligro de extinción de las Islas Galápagos.

\begin{tabular}{|c|c|c|}
\hline Nombre. & Descripción. & Grafico. \\
\hline $\begin{array}{c}\text { Mangles (Rhizophora } \\
\text { mangle) }\end{array}$ & $\begin{array}{l}\text { Es un arbusto o árbol } \\
\text { que varía en su tamaño } \\
\text { y coloración } \\
\text { dependiendo de la } \\
\text { especie, en las islas se } \\
\text { puede encontrar cuatro } \\
\text { tipos de mangle como } \\
\text { el rojo, blanco, negro y } \\
\text { botón. }\end{array}$ & Mangles de Galápagos \\
\hline $\begin{array}{c}\text { Arrayancillo (Maytenus } \\
\text { octágona) }\end{array}$ & $\begin{array}{l}\text { Es un arbusto cuya } \\
\text { corteza es de color gris } \\
\text { oscuro, posee hojas } \\
\text { generalmente gruesas } \\
\text { de forma irregular, sus } \\
\text { flores son verdes y } \\
\text { pequeñas, su fruto tiene }\end{array}$ & $\begin{array}{c}\text { Arrayancillo de } \\
\text { Galápagos }\end{array}$ \\
\hline
\end{tabular}




\begin{tabular}{|c|c|c|}
\hline & $\begin{array}{l}\text { la forma de una } \\
\text { cápsula, } \\
\text { preferentemente cerca } \\
\text { de las playas y zonas } \\
\text { muy secas ya que es } \\
\text { muy resistente a épocas } \\
\text { de sequía. }\end{array}$ & \\
\hline $\begin{array}{c}\text { Uña de Gato } \\
\text { (Zanthoxylumfagara) }\end{array}$ & $\begin{array}{l}\text { Es un arbusto que } \\
\text { puede llegar a medir } \\
10 \mathrm{~m} \text { de altura, sus } \\
\text { ramas posen espinas } \\
\text { fuertemente } \\
\text { enganchadas, posee un } \\
\text { fruto de color negro } \\
\text { azulado. }\end{array}$ & $\begin{array}{l}\text { Uña de Gato de } \\
\text { Galápagos }\end{array}$ \\
\hline $\begin{array}{c}\text { Palo Santo } \\
\text { (Burseragraveolens) }\end{array}$ & $\begin{array}{l}\text { Es un árbol que puede } \\
\text { alcanzar hasta los } \\
12 \mathrm{~m} \text {, el tronco y las } \\
\text { ramas } \\
\text { generalmente de color } \\
\text { gris, posee flores de } \\
\text { color crema y sus } \\
\text { frutos son como } \\
\text { pequeñas cerezas, los } \\
\text { cuales sirven de } \\
\text { alimento a algunas } \\
\text { especies de animales } \\
\text { de las zonas secas, su }\end{array}$ & Palo santo de Galápagos \\
\hline
\end{tabular}




\begin{tabular}{|c|c|c|}
\hline & $\begin{array}{l}\text { nombre se debe que es } \\
\text { un árbol que tiene la } \\
\text { costumbre de florecer. }\end{array}$ & \\
\hline $\begin{array}{c}\text { Cactos } \\
\text { (Brachycereusnesioticus) }\end{array}$ & $\begin{array}{l}\text { Es una planta que } \\
\text { crece en las zonas } \\
\text { áridas de las islas, son } \\
\text { capaces de almacenar } \\
\text { en su tallo grandes } \\
\text { cantidades de agua } \\
\text { para así sobrevivir a } \\
\text { las épocas de sequía, } \\
\text { es característicamente } \\
\text { espinoso se dice como } \\
\text { un medio de defensa } \\
\text { para proteger sus } \\
\text { frutos de aquellos } \\
\text { predadores naturales, } \\
\text { en las islas Galápagos. }\end{array}$ & Cactos de Galápagos \\
\hline
\end{tabular}

Nota. - La disminución de la flora endémica del sector podría traer daños al ecosistema. Fuente adaptada Ministerio del Turismo.

\section{Criterio:}

Todas las plantas anteriormente mencionadas son aquellas que están en peligro de extinción no solo por el turismo masivo sino ya sea por la tala indiscriminada que a existido en la zona, o por los mismos animales domésticos que son declarados como una plaga los cuales destruyen no solo la flora sino la fauna y todo esto juntado con el turismo de masa que hay anualmente la demanda de nuevos establecimientos turísticos los que cada año destruyen uno porcentaje de terreno virgen el mismo que podría ser un habitad natural para la flora, el turismo masivo a traído varias consecuencias en la 
flora ya sea por su destrucción o la contaminación que existía en lugares estratégicos para la sobrevivencia de las plantas.

\section{Fauna Endémica.}

Según las investigaciones realizadas por la empresa Ministerio de Turismo (2018) puede decirque los animales en las islas Galápagos son generalment clasificados como especies nativasn endémicas o introducidas, especies endémicas son animales que sólo se encuentran en las islas y en ninguna otra parte del mundo en un hábitad natural.

Se puede considerar como un buen ejemplo es el turismo de naturalza que se esta realzando en la actualidad que no solo atrae a turista nacionales sino a los extranjeros que son los que mas divisas es decir mas dinero invierten en el pais logrando generar empleo y nuevas fuente economicas para el sector, lo que no puede elogiar a gritos es el daño que estan provocado al ecosistema el daño por el turismo masivo que hay en el sector que provoca la muerte de algunos animales por la contamicion del sector.

Figura N 3: La teoría de la evolución

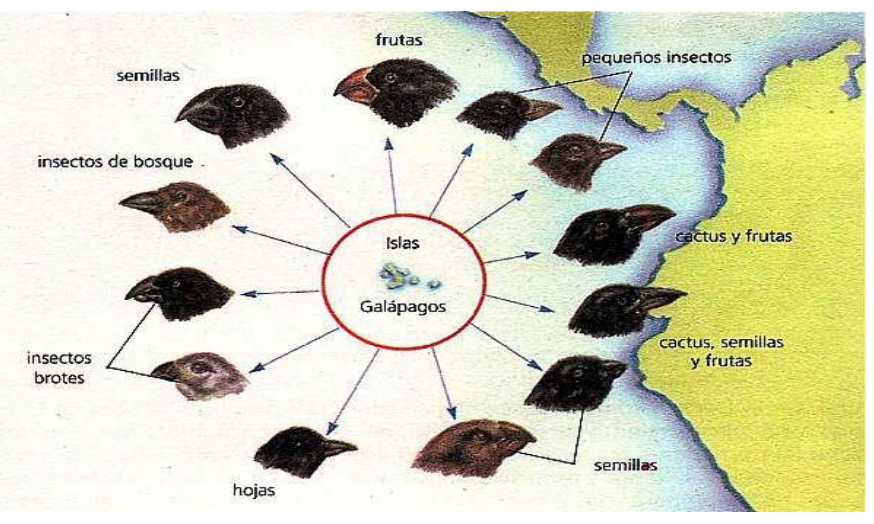

Fuente: Elaboración propia.

\section{Criterio:}

La fauna endeica de las Islas Galápagos es muy importante no solo porque ellas dieron la oportunidad de poder realizar la investigacion de la evolucion por Charles Darwin las que dieron a un paso mas importante, en si la fauna provoca que lleguen miles, millones de turistas anualmete a visitarlas y realizando en turismo de naturales y cientifico o investigativo los que causa que ellos dejen una gran cantidad de dinero al 
momento de ser visitadas y asi poder generar economia local e ingresos alpais por el simple echo de que Galapagos es una de las maravilla naturales y siendo visitada mayor mente por los turistas extranjeron mayor mente por los Estadonudences y Canadiences siendo los que mas gastan por los atractivos que ofrece la isla y no solo por eso sino por conocer ms de cerca a las especies endemicas de las islas tratando de hallar expliv ¿cacion de como en ellas hasy una gran variedad de fauna y biodiversidad que los deja maravilados y con gans de regresar para poder volver a vivir una experiencia innolvidable con grandes sorpresas naturales que ella ofrece.

\section{Clasificación de la fauna en las islas Galapagos}

En las Islas Galápagos podemos encontrar una extensa variedad de especies endémicas las que provocan ser conocidas, lo que causa que anualmente existan barias visitas al sector ya sea por los lugares estratégicamente ubicados para los diferentes tipos de turismo que se pueden realizar en las islas.

\section{Fauna endémica de las Islas Galápagos:}

Tabla N 3. Animales en peligro de extinción de las Islas Galápagos.

\begin{tabular}{|c|c|c|}
\hline Nombre. & Descripción. & Grafico. \\
\hline $\begin{array}{l}\text { Iguana } \\
\text { marina }\end{array}$ & $\begin{array}{l}\text { Es una especie endémica de las islas } \\
\text { Galápagos, este reptil tiene la capacidad } \\
\text { exclusiva en el mundo de vivir y cazar } \\
\text { alimento en el mar, animal de sangre fría } \\
\text { su color es muy oscuro. }\end{array}$ & Iguana Marina \\
\hline $\begin{array}{l}\text { Tiburón de } \\
\text { Galápagos }\end{array}$ & $\begin{array}{l}\text { El tiburón de Galápagos es un aficionado } \\
\text { de las aguas de las Islas Galápagos. Por } \\
\text { tal razón, es común observarles en los } \\
\text { ambientes de arrecifes claros, en donde } \\
\text { abundan algunas especies de tiburones. } \\
\text { Por otro lado, el tiburón de Galápagos }\end{array}$ & Tiburón de Galápagos \\
\hline
\end{tabular}




\begin{tabular}{|c|c|c|}
\hline & $\begin{array}{l}\text { puede crecer hasta los } 3,7 \text { metros (12 } \\
\text { pies), y se encuentran comúnmente } \\
\text { distribuidos en grandes grupos. }\end{array}$ & \\
\hline $\begin{array}{c}\text { Pingüino } \\
\text { de } \\
\text { Galápagos }\end{array}$ & $\begin{array}{l}\text { El pingüino de Galápagos es un pequeño } \\
\text { pingüino endémico de las Islas } \\
\text { Galápagos, es mundialmente conocido } \\
\text { por ser el único pingüino capaz de vivir, } \\
\text { de una manera natural, en hábitats al } \\
\text { norte de la línea ecuatorial, esto es } \\
\text { posible, en gran medida, gracias a las } \\
\text { bajas temperaturas que resultan de las } \\
\text { aguas frías de la Corriente de Humboldt } \\
\text { y de la Corriente de Cromwell. }\end{array}$ & Pingüino de Galápagos \\
\hline $\begin{array}{l}\text { Tiburón } \\
\text { cabeza de } \\
\text { martillo }\end{array}$ & $\begin{array}{l}\text { Los tiburones martillo son nativos de las } \\
\text { Islas Galápagos. Sin embargo, también } \\
\text { pueden ser encontrados en muchas } \\
\text { otras partes del mundo, estos tiburones } \\
\text { son mundialmente conocidos por la } \\
\text { forma extraña de su cabeza. }\end{array}$ & $\begin{array}{c}\text { Tiburón cabeza de } \\
\text { martillo }\end{array}$ \\
\hline $\begin{array}{c}\text { Tortuga de } \\
\text { mar }\end{array}$ & $\begin{array}{l}\text { La tortuga verde de Galápagos es una } \\
\text { subespecie de la tortuga de mar verde } \\
\text { y es endémico de las aguas del Océano } \\
\text { Pacífico, y es a menudo llamada } \\
\text { la "tortuga de mar negro", debido a su } \\
\text { color oscuro. Son las únicas tortugas }\end{array}$ & a de mar \\
\hline
\end{tabular}




\begin{tabular}{|l|l|l|l|}
\hline & $\begin{array}{l}\text { marinas para anidar en las } \\
\text { Islas Galápagos. }\end{array}$ & \\
& & \\
\hline
\end{tabular}

Nota. - La fauna está siendo perjudicada por la culpa del ser humano.

Fuente: Ministerio del Turismo.

\section{Criterio:}

Tabla N 4: Clase de turismo que se realiza en las Islas Galápagos del Ecuador.

\begin{tabular}{|c|c|c|}
\hline Nombre. & Descripción. & Grafico. \\
\hline $\begin{array}{l}\text { Turismo de } \\
\text { Naturaleza }\end{array}$ & $\begin{array}{l}\text { El archipiélago de Galápagos, } \\
\text { considerado uno de los sitios más } \\
\text { prístinos del mundo, constituye el } \\
\text { lugar ideal para disfrutar de la } \\
\text { tranquilidad, este destino ofrece a } \\
\text { sus visitantes espacios naturales } \\
\text { extraordinarios, donde es posible } \\
\text { conectarse con la naturaleza en } \\
\text { este feriado de Semana Santa. }\end{array}$ & $\begin{array}{c}\text { Turismo de naturales } \\
\text { islas Galápagos }\end{array}$ \\
\hline $\begin{array}{c}\text { Turismo de } \\
\text { Aventura }\end{array}$ & $\begin{array}{l}\text { Los programas de aventura en } \\
\text { Galápagos son tours múltiples } \\
\text { deportes por tierra especialmente } \\
\text { diseñada para deportistas y } \\
\text { amantes dela aventura. Estos } \\
\text { itinerarios son súper activos, e } \\
\text { incluyen actividades múltiples } \\
\text { deportes como kayak, ciclismo, } \\
\text { senderismo y snorkel mientas } \\
\text { conoces las Islas Galápagos (turismo, } \\
\text { 2018). }\end{array}$ & $\begin{array}{c}\text { Turismo de aventura } \\
\text { islas Galápagos }\end{array}$ \\
\hline
\end{tabular}


ISSN: 2600-5859

\begin{tabular}{|c|c|c|}
\hline $\begin{array}{l}\text { Turismo de } \\
\text { sol y playa }\end{array}$ & $\begin{array}{l}\text { Es que se da en la zona litoral se da en } \\
\text { localidades costeras en las que se } \\
\text { encuentran playas y la mayoría de } \\
\text { tiempo, las condiciones climáticas son } \\
\text { de tiempo soleado y temperaturas } \\
\text { suaves (de } 25 \text { a } 30{ }^{\circ} \mathrm{C} \text { ). En estas } \\
\text { localidades suele haber gran cantidad de } \\
\text { hoteles y actividades para ocio y tiempo } \\
\text { libre. }\end{array}$ & $\begin{array}{l}\text { Turismo de sol y playa } \\
\text { en las islas Galápagos }\end{array}$ \\
\hline $\begin{array}{c}\text { Turismo } \\
\text { Gastronómico }\end{array}$ & $\begin{array}{l}\text { Las diversas etnias ecuatorianas y la } \\
\text { influencia de alemanes, americanos, } \\
\text { noruegos y otras culturas extranjeras } \\
\text { que colonizaron Galápagos, hicieron } \\
\text { que la gastronomía de las islas sea una } \\
\text { fusión de sabores, capaz de sorprender a } \\
\text { los paladares más exigentes. }\end{array}$ & $\begin{array}{c}\text { Turismo gastronómico } \\
\text { en Galápagos. }\end{array}$ \\
\hline $\begin{array}{l}\text { Turismo } \\
\text { Científico }\end{array}$ & $\begin{array}{l}\text { Alrededor de } 120 \text { especialistas en } \\
\text { astronomía, de diferentes partes del } \\
\text { Globo Terráqueo, disfrutaron de los } \\
\text { atractivos turísticos que tienen las Islas } \\
\text { Galápagos; en el marco de la Congreso } \\
\text { Internacional “Origen y Evolución de } \\
\text { los Halos Galácticos Bariónicos”, que se } \\
\text { desarrolla en la en la ciudad de Puerto } \\
\text { Ayora, Isla Santa Cruz, Galápagos. }\end{array}$ & $\begin{array}{c}\text { Turismo científico en } \\
\text { Galápagos }\end{array}$ \\
\hline
\end{tabular}

Nota. - Se puede demostrar que el turismo es una potencia economía en las Islas Galápagos.

Fuente: Ministerio del Turismo. 


\section{Criterio:}

El turismo de naturales es uno de los más importantes en las islas Galápagos ya que se realiza por medio de la flora y de la fauna las cuales en su mayoría atraen a la gente ya sea por la observación de aves, reptiles, mamíferos o por una simple caminata en medio de la naturales o de sus reserva acuáticas las que atraer mucho a los turistas, el turismo de aventura es uno de los más llamativos en el sector ya que no solo se pueden realizarse en tierra sino también en aire y agua los cuales generan gran economía en el sector y por ende en el país siendo unos de los más solicitados por visitantes nacionales o extranjeros ya que el llegar al extremo o tener algo de riesgo es lo que le hace llamativo por experimentar unos minutos con adrenalina y llenarse de experiencias nuevas ,el turismo de sol y playa es uno de los más importantes no solo a nivel nacional sino internacional por lo que varios hoteles grandes se han ubicado en puntos estratégicos del mundo y uno de ellos son las islas Galápagos del Ecuador en las que grandes hoteles ofrecen no solo hospedaje sino alimentación es decir que han sacado grandes ventajas de los recursos naturales convirtiendo en uno de los punto más grandes y llamativos a nivel de turismo de sol y playa, el turismo gastronómico es aquel en el que las personas realizan recorridos gastronómicos y van visitando los mejores restaurantes de las zona pero en especial los platos típicos del sector y por el final es el turismo científico es el más importante se puede decir porque es aquel que se dio desde que Charles Darwin comenzó sus investigaciones.

\section{Conclusiones}

- Las islas Galápagos del Ecuador es un gran fuente de dinero de lo cual los habitantes han comenzado aprovecharlo y generar trabajo para los mismo lo cual ha sido beneficio para ellos y los turistas ya sea por las infraestructura y buen trato de la gente.

- Los diferentes atractivos turísticos dan las islas el renombre que estas tienen con una maravillosa flora y fauna endémica causa motivación a conocerlas y llenarse de experiencias inolvidables.

- A la solución que se pudo llegar es conocer los lados positivos que sería la ganancia de divisas para el sector y el país en general y negativos la contaminación que se 
estaba generando o por el turismo de masas la destrucción de sectores protegidos en las islas Galápagos del Ecuador.

- Actualmente se puede considerar a las islas Galápagos del Ecuador como una de las maravillas naturales más importantes del mundo por toda la flora y fauna endémica que la misma tiene y que ya ha estado creando leyes para que esto siga siendo, como a las personas que voten basura para provocar contaminación en la área protegida será multado con un porcentaje de dinero.

\section{Referencias bibliográficas.}

Alan Tye. (2006). Libro Rojo dela plantas endémicas del Ecuador. Obtenido de Bioweb : https://bioweb.bio/floraweb/librorojo/galapagos/

Alvarado, A. (09 de 03 de 2016). Ecuador: personajes y especies (Galápagos y Continente). Obtenido de PLANTAS DE GALÁPAGOS: http://dspace.espoch.edu.ec/bitstream/123456789/2192/1/23T0332\%20.pdf

Amador, E. (1996). Servicio Parque Nacional Galápagos Instituto Ecuatoriano Forestal y de Áreas Naturales y Vida Silvestre . Obtenido de Puerto Ayora, Islas Galápagos: http://files.admonturistica.webnode.com.co/20000006781c3882bf6/Capacidad\%20de\%20carga.pdf

Arias, I. A. (12 de 2004 ). Lyonia . Obtenido de Lyonia, Volume 6(2), http://www.lyonia.org/articles/rbussmann/article_262/pdf/articleBody.pdf

Barriga, A. M. (6 de 10 de 2014). Planificación y Gestión del Turismo. Obtenido de La contradicción del turismo en la conservación y el desarrollo en Galápagos: http://170.210.83.98:8080/jspui/handle/123456789/441

Captur. (2018). Fragata Yacht. Obtenido de Fragata Yacht: http://www.yatefragata.com/index.php/islas-galapagos/animales-de-las-islasgalapagos.html

Epler, B. (2007). Turismo, Economía, Crecimiento Poblacioal y Concervación en Galápagos. Obtenido de Turismo, Economía, Crecimiento Poblacioal y Concervación en Galápagos: http://edpcollege.info/ebooks-pdf/Informe_Turismo-Epler-es_5-08.pdf

Gómez, S. (2015). Noray. Obtenido de Noray: https://www.noray.com/blog/el-turismo-desol-y-playa-mas-vigente-que-nunca/

Grenier, C. (2007). Conservación contra natura las islas Galapagós . Lima: Universidad Andina Simon Bolivar . 
Ministerio de Turismo . (2018). Fragata Yacht. Obtenido de Fragata Yacht: http://www.yatefragata.com/index.php/islas-galapagos/animales-de-las-islasgalapagos.html

Muñoz, A. (2015 ). La contradicción del turismo en la conservación y el desarrollo en Galápagos - Ecuador. Ciudad Autónoma de Buenos Aires : Estudios y perspectivas en turismo .

Place, P. (28 de 04 de 2017). Galapagos Conservation. Obtenido de Galapagos Conservation: http://descubriendogalapagos.ec/descubre/ecodesarrollo/principios-de-lasostenibilidad/una-economia-sostenible/

Turismo, M. d. (16 de 03 de 2017). Ministerio del Turismo. Obtenido de Ministerio del Turismo: https://www.turismo.gob.ec/turismo-cientifico-una-alternativa-para-lasislas-galapagos/

Turismo, M. d. (27 de 12 de 2017). Ministerio del Turismo . Obtenido de Ministerio del Turismo : https://www.turismo.gob.ec/la-gastronomia-de-galapagos-deleita-lospaladares-de-los-turistas/

Turismo, M. d. (21 de 3 de 2018). Galápagos, un destino para disfrutar la naturaleza en este feriado. Obtenido de Galápagos, un destino para disfrutar la naturaleza en este feriado: https://www.turismo.gob.ec/galapagos-un-destino-para-disfrutar-lanaturaleza-en-este-feriado/

turismo, M. d. (2018). Nature Galapagos \& Ecuador. Obtenido de Nature Galapagos \& Ecuador: https://naturegalapagos.com/es/

Zhirzhan, S. B. (10 de 07 de 2012). OCTAVO CIENCIAS NATURALES. Obtenido de Flora Endemica de las Islas Galapagos: http://octavoccnn2012.educationspot.com/p/floraendemica-de-las-islas-galapagos.html.

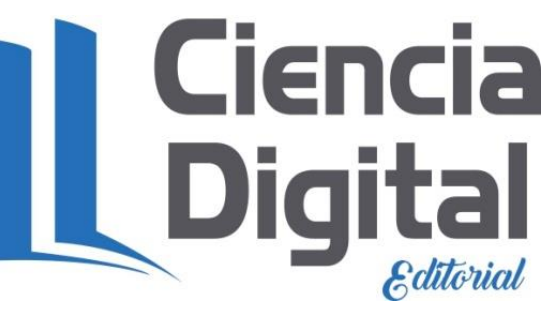


Para citar el artículo indexado.

Velasteguí López, P. (2019). Las Islas Galápagos del Ecuador Como Potencia Turística. ConcienciaDigital, 2(1), 6-23. https://doi.org/10.33262/concienciadigital.v2i1.926

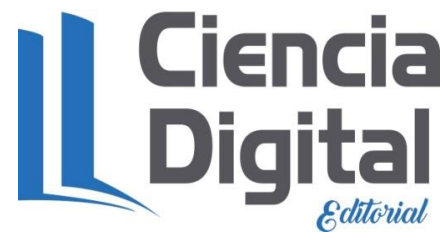

El artículo que se publica es de exclusiva responsabilidad de los autores y no necesariamente reflejan el pensamiento de la Revista Conciencia Digital.

El articulo queda en propiedad de la revista y, por tanto, su publicación parcial y/o total en otro medio tiene que ser autorizado por el director de la Revista Conciencia Digital.

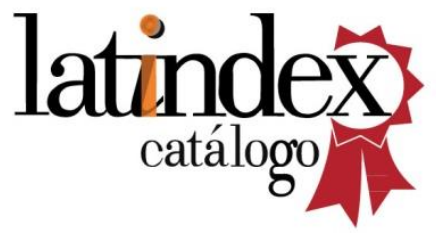

Conciencia

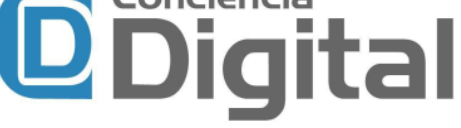

